\title{
Yves Ledure, La rupture. Christianisme et modernité
}

Paris, Ếditions Lethielleux, 2010, 202 p.

Isabelle Jonveaux

\section{(2) OpenEdition}

\section{Édition électronique}

URL : http://journals.openedition.org/assr/22909

DOI : $10.4000 /$ assr.22909

ISSN : $1777-5825$

\section{Éditeur}

Éditions de l'EHESS

Édition imprimée

Date de publication : 31 décembre 2011

Pagination : 192

ISBN : 9782713223273

ISSN : 0335-5985

\section{Référence électronique}

Isabelle Jonveaux, «Yves Ledure, La rupture. Christianisme et modernité », Archives de sciences sociales des religions [En ligne], 156 | octobre-décembre 2011, document 156-64, mis en ligne le 15 février 2012, consulté le 21 septembre 2020. URL : http://journals.openedition.org/assr/22909 ; DOI : https://doi.org/10.4000/assr.22909

Ce document a été généré automatiquement le 21 septembre 2020

(c) Archives de sciences sociales des religions 


\title{
Yves Ledure, La rupture.
} Christianisme et modernité

Paris, Ếditions Lethielleux, 2010, 202 p.

\author{
Isabelle Jonveaux
}

\section{RÉFÉRENCE}

Yves LEDURE, La rupture. Christianisme et modernité, Paris, Ếditions Lethielleux, 2010, $202 \mathrm{p}$.

1 Dans le contexte du débat autour du Memorandum des théologiens allemands («Kirche 2011, Ein notwendiger Aufbruch ", manifeste signé par plus de deux cents professeurs de théologie dans la Süddeutsche Zeitung réclamant des réformes de la part de l'Église sur participation des laïss, mariage des prêtres, ordination des femmes, accueil des divorcés remariés, etc.), l'ouvrage d'Yves Ledure prend un écho tout particulier. Livre de philosophie, essai ou franc manifeste pour une réforme de l'Église - ou plus exactement du christianisme -, cet ouvrage est difficilement classable. Plus que le besoin d'une réforme institutionnelle, c'est celui d'une relecture du système de croyance auquel l'auteur engage le lecteur mais aussi sans doute l'Église elle-même.

Plus de deux siècles après l'avènement des Lumières et de l'Aufklärung, Y. Ledure part du constat que le christianisme n'a toujours pas pacifié ses relations avec la modernité et, surtout, ne l'a toujours pas intégrée dans son mode de fonctionnent. La crise d'autorité de l'Église actuelle serait selon lui due à ce décalage irréductible entre une religion demeurée identique à son origine et un monde dont les référentiels ont été profondément modifiés. L'auteur oriente alors son projecteur dans trois directions principales : celle de l'institution, celle du corps et de l'incarnation, et enfin, celle des rapports entre philosophie et théologie, incluant la question de la transcendance.

3 Ce qu'identifie Y. Ledure dans le rapport du catholicisme à la modernité est rien moins qu'une véritable rupture comme l'annonce le titre. Un christianisme qui serait resté crispé sur ses caractéristiques vieilles de deux mille ans et marquées par un contexte 
historico-social désormais lointain. L'environnement actuel est celui de la sécularisation qui conteste le christianisme comme humanisme, car il ne le conçoit pas comme une voie possible d'épanouissement. Sans doute ce propos serait-il à nuancer car la virtuosité religieuse est de plus en plus considérée comme une possibilité de réalisation personnelle. Néanmoins, l'auteur met en lumière cette rupture créée par la prise en considération d'un humanisme sans Dieu. La question intéressante soulevée par ce chapitre et qui ne manquera pas d'interpeler le sociologue, est celle de la reconnaissance par une religion de ses caractéristiques historiquement marquées, donc humainement déterminées et non pas de manière divine. La reconnaissance de ces éléments ouvrirait sur une possibilité d'actualisation, d'aggiornamento, en discernant les caractéristiques intrinsèquement religieuses, des aléas socioculturels.

4 L'Église - ecclesia - doit effectivement se comprendre non pas comme Royaume déjà advenu et parfait mais comme institution terrestre viciée et imparfaite dans son origine-même. N'est-ce pas sur Pierre, lui qui a cherché plusieurs fois à se mettre en travers du projet de Jésus, que ce dernier a fondé son Église? Cette institution terrestre, comme toute institution, est nécessairement une création historique, ancrée dans un contexte social déterminé, appelé donc à changer avec le temps. «L'institutionnel, nécessairement d'ordre historique, ne sera jamais le miroir du mystère, ne se traduit pas en termes mystiques.» (p. 52) L'Église est donc une institution humaine destinée à évoluer avec l'humain. Le pouvoir absolu de la curie romaine, héritée du système impérial sur lequel l'Église naissante a calqué son fonctionnement, ne s'accorde plus avec l'ère démocratique de la modernité. « Il faudrait donc que, d'une façon ou d'une autre, l'ecclesia conjugue sa forme hiérarchique avec l'exigence de liberté démocratique.» (p.62) Selon lui, l'Église n'intègrerait pas assez l'idée de liberté, ne laissant donc pas assez de place au libre-arbitre humain.

Un point essentiel de la modernité sur lequel le christianisme peine à s'adapter est en effet celui, selon Y. Ledure, du «tournant anthropologique» (p. 67). Le christianisme est cette religion particulière, et, en cela, distinct du judaïsme ou de l'islam, qui se fonde sur la notion d'incarnation et de ce que Y. Ledure appelle l'« anthropothéisme ", c'est-à-dire, que "le divin y garde la résonnance transcendante, à ceci près qu'il s'ouvre à un échange avec l'homme»(p.70). Cette incarnation ouvre un nouveau chemin de réalisation pour l'homme qui ne s'arrêtera pas avec la mort. Le christianisme serait unique, les relations entre l'homme et Dieu se définissant comme "filialité », " où l'initiative revient à celui qui se reconnaît fils » (p. 72). Aucunement conflictuelle, cette relation se veut réciproque et peut parfaitement intégrer l'autonomie de l'homme, ce que, selon l'auteur, le christianisme ne prend pas suffisamment en compte, risquant par là de ne plus être un "agent culturel significatif» (p. 87) dans la modernité. Ne sachant pas, ou ne voulant pas, accepter la nouvelle définition de l'homme, autonome et porteur d'un inconscient, le christianisme pourrait se trouver dans ce qu'Yves Ledure appelle un « trou d'air » (p. 88).

6 L'homme devient donc le centre de toute compréhension dans l'ère moderne. Et cet homme est sexué. Or la sexualité détermine tout rapport à l'existence. Non pas la génitalité, cette recherche du plaisir génital, mais comme corps différencié : hommes et femmes. Le christianisme antique se serait fondé sur les pierres de la philosophie antique qui ne définissait pas l'homme par son corps mais pas son esprit. Sans doute faudrait-il encore nuancer l'accusation d'Y. Ledure soutenant que l'Église s'adresse à l'homme comme si c'était toujours celui de l'Antiquité. Il faudrait aussi rappeler la plus 
grande prise en considération du corps dans le christianisme ancien, qui se démarque par là des philosophies grecques et latine; les condamnations du corps ne sont venues que peu à peu, prenant principalement naissance dans le monachisme ascétique. Définir l'homme à partir de sa rationalité occulte sa corporéité. Or la relation au divin définie par le christianisme pose comme postulat cette qualification de l'humain et n'entre donc plus en résonnance avec un homme centré sur sa corporéité. À cette occasion, Y. Ledure se demande "comment [le célibat sacerdotal ou consacré] peut intégrer la dimension sexuelle propre à toute existence humaine » (p. 109), en écho là encore aux questions soulevées par le Memorandum allemand. La question de la prise en compte de la dimension sexuée de l'homme pose aussi celle de la place réservée à la femme dans l'institution ecclésiale et dans la théologie chrétienne en générale. Dans cette dernière, selon l'auteur, le masculin est le référentiel humain sans lequel la femme ne se définit pas, vision largement héritée de la société patriarcale dans laquelle s'est développé le christianisme. Ce dernier aurait alors un rôle essentiel à jouer dans la modernité en ressaisissant la complémentarité et l'égalité fondamentales de l'homme et de la femme appelés chacun à " devenir des êtres humains singuliers » (p. 125). Alors que la société elle-même peine à intégrer concrètement l'égalité de l'homme et de la femme dans leur identité différente, le christianisme pourrait au contraire se faire porteur d'un message inventif pour le Xxi ${ }^{\mathrm{e}}$ siècle.

7 Le dernier point étudié par Y. Ledure concerne les rapports entre théologie et philosophie ainsi que ceux entre la transcendance et la finitude. Le siècle des Lumières a consommé la rupture entre la théologie et la philosophie qui, auparavant, collaboraient étroitement pour construire une vision du monde. La sécularisation se fait le lieu d'un discours autonome par rapport à un transcendant. Il n'y a alors plus d'unité dans l'explication proposée de l'univers, mais le brouillage d'une "vérité morcelée» (p. 148). L'auteur soutient que le christianisme n'a toujours pas reconnu cette séparation de fait entre la théologie et la philosophie, qui se traduit en terme d'autonomisation de la philosophie. À cela s'ajoute un effacement de la notion de transcendance, et l'individu de la modernité a plus le souci de soi que celui du divin, comme le soutient Danièle Hervieu-Léger (Catholicisme, la fin d'un monde, Bayard, 2003, p. 132) parlant de la recherche d'une « religion qui fait du bien». La transcendance disparue, c'est tout le cadre de significations pour l'homme qui s'effondre. Sans porter à l'idéalisme, la transcendance permettrait à l'homme « d'entreprendre une aventure différente de celle des autres êtres vivants, une aventure non seulement de jouissance de l'originaire vital, mais de dépassement et de transformation d'un initial marqué, fragilisé par la finitude.» (p. 164). Le christianisme devrait alors plus tenir compte de cette tension fondamentale de l'homme, entre sa finitude déterminante d'une part et son désir et sa capacité de dépassement d'autre part plutôt que de demeurer dans un « idéalisme désespérant» (p. 168). Mais cette réintégration de la transcendance ne doit pas se faire une « humanisation du divin » (p. 174) selon l'auteur qui s'oppose en cela au philosophe Luc Ferry, car le divin se dissout dans une trop grande proximité avec l'homme.

8 Finalement, l'homme serait-il fatigué de Dieu ? C'est cette question que pose Y. Ledure dans sa conclusion, en miroir de la «fatigue d'être soi » décrite par A. Ehrenberg. La transcendance, loin d'être une libération comme le voudrait le christianisme, pèse sur les individus comme contrainte supplémentaire. «L'homme de la modernité est fatigué de Dieu car il est fatigué de lui-même.» (p. 197) Mais le christianisme est une religion humaniste essentiellement communautaire qui cherche à s'opposer à l'individualisme 
ambiant. Et c'est cela dont aurait besoin l'homme d'aujourd'hui selon Ledure, d'où la nécessité pour le christianisme de retrouver son incarnation première et libératrice.

Si certaines de ses affirmations, notamment sur le christianisme des origines seraient à nuancer, on ne peut qu'acquiescer à sa fine analyse du déplacement du référentiel divin au référentiel humain, et plus précisément du référentiel de l'esprit au référentiel du corps. «L'homme de la modernité se comprend à partir de son corps.» (p. 104) Sans doute est-ce là le cœur de sa démonstration et ce qui a renforcé ma conviction personnelle de l'intérêt pour la sociologie des religions de se pencher sur la question du corps pour comprendre le religieux moderne.

C'est sans mâcher ses mots et sans retenir certaines accusations qui dénotent sans doute une certaine déception face au christianisme tel qu'il est aujourd'hui, qu'Y. Ledure appelle à une réforme, non pas simplement institutionnelle, ni dogmatique, mais qui serait un changement de regard, de référentiel dans la vision du monde, des hommes et de Dieu. Plus profondément que des évolutions concrètes, c'est la théologie chrétienne, dans ce qu'elle offre de Dieu aux hommes, qui devrait se renouveler en profondeur selon lui.

11 Cet ouvrage reflète donc de manière assez troublante les questions soulevées dans le Memorandum des professeurs germanophones de théologie, comme s'il se faisait un miroir fidèle de l'air du temps. Il serait alors un complément intéressant pour comprendre le fondement philosophique et théologique de certains axes de réformes proposés par ce manifeste. La question de la participation des fidèles rejoint effectivement celle de la dimension démocratique de l'Église, quand celle de la liberté de conscience fait écho à la revendication de l'auteur d'accorder plus de place au librearbitre. Naturellement, comme je l'ai déjà mentionné, les propos autour du mariage des prêtres et de l'accès des femmes au sacerdoce, s'ancrent dans le changement de paradigme dans la définition de l'homme, passant du référentiel de l'esprit au référentiel du corps. Mais qu'attend l'auteur des exhortations qu'il lance plus à l'Église qu'au lecteur? Espère-t-il cette réforme profonde du système religieux chrétien? Espère-t-il ce décalage de l'axe sur lequel il tourne depuis deux mille ans? Mais il ne s'agit pas ici d'une polémique sur des questions récurrentes du christianisme moderne, mariage des prêtres ou sacerdoce des femmes, il est aussi un plaidoyer pour un christianisme nouveau et une défense du christianisme comme capacité à s'adapter à la modernité et à conserver - s'il accepte de se transformer - une place pertinente dans l'explication du monde moderne. L'intérêt de cet ouvrage est donc de nous proposer un retour aux racines de la « rupture » entre christianisme et modernité, qui montre que les débats institutionnels sont vains s'ils ne prennent pas en compte l'origine de cette incompréhension entre l'Église et une partie des croyants et intellectuels chrétiens.

D'un point de vue sociologique, cet essai est donc un complément intéressant à la vague de questions que soulève actuellement la théologie. Il vient nous prouver, à la suite des remous actuels dans la recherche théologique, le débat de fond auquel l'Église se trouve confrontée. Il se joue ici des questions qui vont bien au-delà d'un simple débat sur un droit ou non au mariage des prêtres pour leur équilibre personnel, mais sur les rapports fondamentaux d'une religion à elle-même dans son évolution temporelle et sa capacité à s'adapter à ces inflexions socio-historiques. Un débat qui nous emmène plus loin que des questions concrètes sur la place de tel ou tel groupe dans l'Église, et auquel la sociologie des religions ne saurait demeurer indifférente. Car en effet, la sociologie a 
trop peu pris en compte les évolutions de l'intérieur même du système de croyance qui détermine tout : pratiques, rituels et autres institutions. 